\title{
Pneumothorax Complicating Port-a-Cath and Groshong Catheter Positioning in Children: Our Experience before Routine Ultrasound-Guided Puncture*
}

\author{
Silvia Guenzani ${ }^{1,2 \#}$, Paola Previtali ${ }^{2}$, Federico Piccioni ${ }^{2}$, Maria Chiara Allemano ${ }^{2}$, Serena Catania ${ }^{3}$, \\ Martin Langer ${ }^{1,2}$
}

${ }^{1}$ Department of Pathophysiology and Transplantation, University of Milan, Milan, Italy; ${ }^{2}$ Department of Anesthesia, Intensive Care and Palliative Care, Fondazione IRCCS Istituto Nazionale dei Tumori, Milan, Italy; ${ }^{3}$ Pediatric Oncology Unit, Fondazione IRCCS Istituto Nazionale dei Tumori, Milan, Italy.

Email: " silvia.guenzani@gmail.com

Received June $18^{\text {th }}, 2013$; revised July $20^{\text {th }}, 2013$; accepted August $19^{\text {th }}, 2013$

Copyright (C) 2013 Silvia Guenzani et al. This is an open access article distributed under the Creative Commons Attribution License, which permits unrestricted use, distribution, and reproduction in any medium, provided the original work is properly cited.

\begin{abstract}
Objective: To study incidence and management of long term central venous catheter (CVC) placement related pneumothorax (PNX) in children. Aim: To construct a baseline value before the introduction of systematic use of ultrasound guidance, which requires specific training and equipment. Background: Anesthesia Service and Pediatric Oncology of the Italian National Cancer Center; patients were children (age $\leq 18$ years) with solid tumors, needing long-term central venous catheters (Groshong or Port-a-Cath). Materials/Methods: Catheter placement was performed, mostly under general anesthesia, utilizing a micropuncture $5-7$ Fr needle and fluoroscopy. In the study period ultrasound was used only in case of previously failed attempts. Relevant data were collected retrospectively. Results: From August 2008 to December 2011, 452 catheters were implanted to our patients. The prevalent approach was from subclavian vein (left $85.7 \%$, right 9.7\%); in few cases internal jugular vein was chosen (right $2.4 \%$, left $2.2 \%$ ). Pneumothorax occurred in 14 patients $(3.1 \%$; 95\% CI 1.9 - 5.1). In 4/14 children the PNX was considered minimal and not treated. In 10 patients the PNX was drained. In 7 cases a traditional, surgical thoracostomy was performed, while in 3 children a 14-Ga polyurethane catheter (Arrow International ${ }^{\circledR}$ ) was inserted over a wire guide in the pleural space by anaesthetists. Conclusions: In our centre rates of PNX are the same as those described in literature and are expected to lower when ultrasound guidance of the puncture will be routinely applied. Percutaneous drainage of PNX seems as effective as surgically placed thoracostomy catheter, but less invasive.
\end{abstract}

Keywords: Pneumothorax; Central Venous Catheter; Groshong; Port-a-Cath; Children; Drainage

\section{Introduction}

Long term venous access through totally implantable venous access devices (TIVAD), partially implantable tunneled cuffed catheters, or more recently peripherically inserted central venous catheters (PICC) are essential for administering chemotherapy also to young oncologic patients. Positioning of these catheters is reported as a relatively safe procedure with little associated morbidity and nearly no mortality, where this risk is outweighed by

\footnotetext{
*Important Note: Main results of this study have been presented as oral communication at SMART CONFERENCE on May 2012, this is why you can find a similar abstract at this link:

http://www.altrimedia.biz/smart-cd2012/Comunicazioni_Libere/10_TE RAPIA INTENSIVA PEDIATRICA/54_Guenzani.pdf.

"Corresponding author.
}

the benefit obtained [1].

An implanted port is preferred when its use is intermittent (the skin protects the device from infections and rupture) and can remain in situ for years, while an external catheter is commonly used for continuous infusion during a shorter period of time. Percutaneous positioning of catheters can be achieved by various central accesses: through the subclavian vein (SCV), the internal jugular vein (IJV), or, in special cases, the femoral vein, while the brachial vein is preferred for PICC insertion.

Pneumothorax (PNX) is a typical early, mechanical complication of the puncture of the subclavian vein. The SCV approach is more frequently used than the IJV access in small children because the exit site is placed at proper distance from the mouth, it is easy to protect and 
tunneling of the catheter is rather simple and linear. The infraclavicular, landmark based insertion for long term central venous catheters (CVCs) in children is a widely used approach, but ultrasound (US) guidance is difficult. Also in our center catheter placement in pediatric patients under sonographic guidance is still uncommon, and anesthetists usually perform a landmark based puncture of the $\mathrm{SCV}$, with the approach through the IJV as second choice.

In this retrospective study we try to assess the risk of PNX in our experience compared to the literature, in order to discuss/revise our catheter placement policy in children.

\section{Materials and Methods}

Two options for elective long term venous accesses are available at our Hospital: Port-a-Cath (Titanium LowProfile Implantable Port-Bard Access Systems or others), a totally implantable venous access device (TIVAD), and Groshong catheter (Bard Access Systems), a tunneled external silicon-rubber device with a three-position pressure-sensitive valve near the distal tip.

Long term CVC placement in pediatric patients is proposed by pediatric oncologists to the anesthesia service. The preoperative evaluation includes medical history, body examination to evaluate their physical habitus and anatomic pitfalls (obesity, chest tumors or adenopathies of the mediastinum), laboratory exams (complete blood and platelet count, coagulation screening) and a chest X-ray. Cannulation is performed percutaneously in an operating room where anesthesia facilities and $\mathrm{C}$-arm X-ray machine are available.

Left infraclavicular SCV cannulation is the access of first choice. If more then one attempt fails, other sites (IJV) are tried. A micropuncture set (Micropuncture Introducer Set 5.0 - 7.0 Fr-Cook) is used for the puncture of the vein and catheters are inserted under fluoroscopic control. In nearly all pediatric patients the procedure is performed under general, total intravenous anesthesia, mechanical ventilation and standard monitoring. There is no dedicated catheter-team and all staff anesthetists and many residents are actively involved.

A chest X-ray is performed $6-12 \mathrm{hrs}$ after catheter placement to document the location of catheter tip and to rule out mechanical complications like PNX, and hemoor hydrothorax.

In case of evidence of PNX the need for drainage is discussed with a thoracic surgeon and/or a staff-anesthetist, taking into account the clinical symptoms, size and progression of the pneumothorax. Drainage of PNX is performed by the thoracic surgeon with a $16 \mathrm{Ch}$ chest tube drainage, or by the anesthetist with a single-lumen $14 \mathrm{Ga}$ venous catheter (Arrow ${ }^{\circledR}$ International-central venous catheterization set) over a wire guide, after percu- taneous puncture of the pleural space. The choice between chest tube drainage or percutaneous catheter is not standardized yet, but we are getting used to utilize increasingly the less invasive option.

Data analysis was performed using IBM SPSS Statistics v19. Chi-quare test was adopted to compare discrete variables. Normality distribution of continuous variables was verified with Shapiro-Wilk test. T-test for independent groups was used to compare parametric data. A $\mathrm{p}$ value of less than 0.05 was considered as statistically significant.

\section{Results}

From August 2008 to December 2011, 452 long term vascular access devices were implanted in pediatric patients (median age 8 years, range 3 months - 18 years) at our Institute.

In 159 (35.2\%) children a Groshong catheter was chosen and in 293 (64.8\%) a TIVAD. Infraclavicular SCV cannulation was in all cases the access of first choice, followed by puncture of IJV when more than one attempt failed. The procedure was performed in $93 \%$ of cases under general anesthesia, and in $7 \%$ of cases with local anesthesia. The final catheterization site was primarly the subclavian vein (left SCV 85.7\%, right SCV 9.7\%, right IJV 2.4\%, left IJV 2.2\%). Anatomic landmark oriented puncture was the first choice technique in all patients, US guidance after initial failed attempts was performed in $2 \%$ of the procedures.

PNX was diagnosed at the control chest X-ray in 14 cases $(3.1 \%$; $95 \%$ CI 1.9 - 5.1) always after several failed punctures of the SCV. See Table 1 for patients' demographic data. Ten of these patients underwent PNX drainage, in 4 cases the PNX was minimal, not requiring any treatment. Seven patients underwent a $16 \mathrm{Ch}$ tube thoracostomy, while in 3 cases the pleural air was drained by a single-lumen $14 \mathrm{Ga}$ venous catheter (Arrow ${ }^{\circledR}$ International-central venous catheterization set). In the latter approach air was aspired by a syringe once or twice a day with the catheter locked during the intervals. Chest tubes and "chest catheters" remained in situ for a variable period between 2 and 12 days (average: catheters for 4.3 days, chest tubes for 5.6 days; $p=0.633$ ). No other early complications or long term consequences were recorded.

\section{Discussion}

In our study the rate of pneumothorax (3.1\%; 95\%CI 1.9 - 5.1) after a central venous catheter insertion by puncture of the SCV (infraclavicular approach) was in the higher range, compared to the pooled data (Table 2 and Figure 1) from 7 pertinent studies in 2839 children $(1.7 \%$; $95 \%$ CI $1.3-2.3)$ [2-8]. The $1.4 \%$ difference is 
Children: Our Experience before Routine Ultrasound-Guided Puncture

Table 1. Demographic data of patients with PNX (DT, days of thoracostomy; US, ultrasound; SB, spontaneous breathing and local anesthesia; MV, mechanical ventilation and general anesthesia; SCV, subclavian vein; PNET, primitive neuroectodermal tumor; MPNST, malignal peripheral nerve sheath tumor; NHL, non Hodgkin lymphoma).

\begin{tabular}{|c|c|c|c|c|c|c|c|c|}
\hline $\mathrm{PT} \mathrm{N}^{\circ}$ & Sex & Age & Diagnosis & DT & Site of catheterization & US & $\mathrm{SB}$ or MV & Procedure \\
\hline 1 & M & 16 & Hepatoblastoma & 4 & Left SCV & NO & MV & Tube thoracostomy \\
\hline 2 & M & 12 & Medulloblastoma & l & Left SCV & NO & MV & None \\
\hline 3 & M & 10 & Glioblastoma & 5 & Left SCV & NO & MV & One-lumen 14Ga Arrow \\
\hline 4 & M & 14 & Lynfoprolipherative disease & / & Left SCV & NO & MV & None \\
\hline 5 & $\mathrm{~F}$ & 14 & Desmoid fibromatosis & 12 & Left SCV & NO & SB & Tube thoracostomy \\
\hline 6 & M & 11 & Glioblastoma & 6 & Left SCV & YES & MV & Tube thoracostomy \\
\hline 7 & $\mathrm{~F}$ & 14 & Intracranic fibrosarcoma & 3 & Left SCV & NO & MV & Tube thoracostomy \\
\hline 8 & M & 17 & PNET & 2 & Left SCV & NO & MV & Tube thoracostomy \\
\hline 9 & $\mathrm{~F}$ & 3 & Neuroblastoma & 2 & Left SCV & NO & MV & Tube thoracostomy \\
\hline 10 & M & 15 & MPNST & / & Left SCV & NO & MV & None \\
\hline 11 & M & 18 & Mediastinal sarcoma & / & Left SCV & NO & MV & None \\
\hline 12 & $\mathrm{~F}$ & 1 & Rabdomiosarcoma & 2 & Left SCV & YES & MV & One-lumen 14Ga Arrow \\
\hline 13 & M & 16 & Troncoencephalic glioma & 10 & Left SCV & NO & SB & Tube thoracostomy \\
\hline 14 & M & 9 & NHL & 6 & Left SCV & NO & MV & One-lumen $14 \mathrm{Ga}$ Arrow \\
\hline
\end{tabular}

Table 2. Incidence data from the literature concerning pneumothorax (PNX) after central venous catheter (CVC) positioning through the SCV. The $95 \% \mathrm{CI}$ are calculated ex post.

\begin{tabular}{|c|c|c|c|c|}
\hline & $\mathrm{N}^{\circ}$ subclavian CVCs & $\mathrm{N}^{\circ} \mathrm{PNX}$ & $\% \mathrm{PNX}$ & $95 \% \mathrm{CI}$ \\
\hline Karapinar et al. 2007 [2] & 119 & 3 & 2.5 & $0.9-7.2$ \\
\hline Araujo et al. 2007 [3] & 197 & 4 & 2.0 & $0.8-5.1$ \\
\hline Citak et al. 2002 [4] & 148 & 2 & 1.4 & $0.4-4.8$ \\
\hline Casado-Flores 2001 [5] & 235 & 6 & 2.6 & $1.2-5.5$ \\
\hline Lefrant et al. 2002 [6] & 707 & 22 & 3.1 & $2.1-4.7$ \\
\hline Casado-Flores 1991 [7] & 322 & 6 & 1.9 & $0.9-4.0$ \\
\hline Johnson et al. 1998 [8] & 1180 & 6 & 0.5 & $0.2-1.1$ \\
\hline Guenzani et al. 2013 & 452 & 14 & 3.1 & $1.9-5.1$ \\
\hline Overall & 3360 & 63 & 1.87 & $1.5-2.4$ \\
\hline
\end{tabular}

remarkable but statistically not significant $(\mathrm{p}=0.061)$. Excluding the outstanding good results in the large series of Johnson et al. $(0.5 \%$; $95 \%$ CI $0.2-1.1)$, the mean incidence of PNX during cannulation of SCV in 2180 children, from the remaining 6 studies, is $2.5 \%$ (95\%CI 1.9 3.3).

Pneumothorax complicating landmark-oriented puncture of the SCV remains therefore a real risk of $\mathrm{CVC}$ placement in children. The standard treatment of PNX is the chest tube insertion and connection to a chest drain canister. Of course this is a cumbersome maneuver for sick children, requiring mostly general anesthesia or deep sedation, more or less postoperative pain and bed rest. The attempt to drain pleural air in spontaneously breathing children with a "minimally invasive" drain (a standard central venous catheter, inserted over a wire-guide) was successful when adopted, leading to rapid resolution, also without catheter connection to the drain canister. One or two syringe aspirations per day allowed removing air and checking for new air accumulation. When 


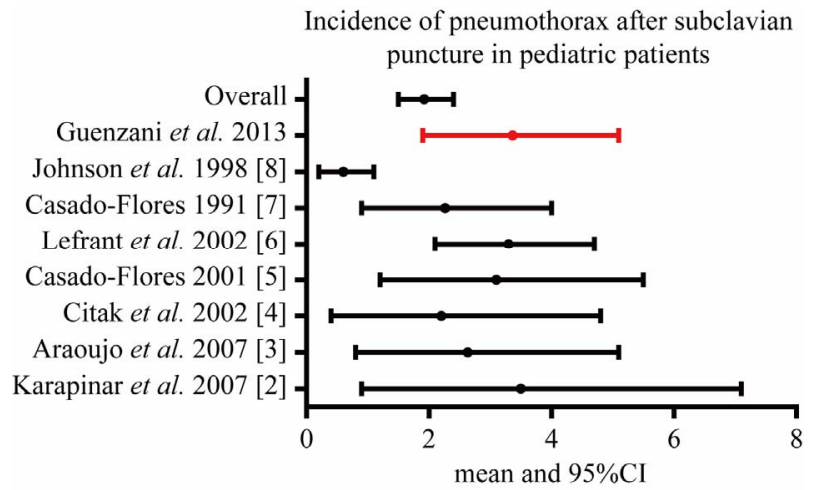

Figure 1. Incidence data from the literature concerning pneumothorax (PNX) after central venous catheter (CVC) positioning through the SCV. The $95 \% \mathrm{CI}$ are calculated ex post.

no more air was aspired for $24 \mathrm{hrs}$ the catheter was removed. The advantage of this approach in children with "non-tension" pneumothorax after accidental pleural puncture is the minimal invasiveness of the procedure and the limited discomfort for the patient.

To achieve the "zero complication" option of CVC insertion, however, a change in our CVC placement policy will be necessary. US guidance techniques have become the gold standard for catheterization of IJV in children. However, IJV catheterization is a difficult procedure in infants, because of anatomy of the region. The SCV approach is preferred in this population. Compared to the classical landmark technique, the US infraclavicular guided cannulation permits puncturing more laterally, reducing not only PNX but also costoclavicular pinch-off complication [9]. Lateral approach is, however, difficult in small children, because of the thinness of the thorax. An ideal alternative, in infants, can be the US supra- clavicular approach [10], with a supraclavicular catheter tunnellization, which offers a good view of the needle and the vein, without any US shadow of the clavicle, and avoids catheter pinch.

\section{Acknowledgements}

The authors and their colleges are grateful to Mariolina Bonalumi, MD who started the long term central venous catheter program for children in our Institute and tutored most of us.

This study is supported by the " $5 \%$ donations" to the Fondazione IRCCS, Istituto Nazionale dei Tumori, Milano.

\section{REFERENCES}

[1] A. Zaghal, M. Khalife, D. Mukherji, et al., "Update on
Totally Implantable Venous Access Devices," Surgical Oncology, Vol. 21, No. 3, 2012, pp. 207-215. http://dx.doi.org/10.1016/j.suronc.2012.02.003

[2] B. Karapinar and A. Cura, "Complications of Central Venous Catheterization in Critically Ill Children," Pediatrics International, Vol. 49, No. 5, 2007, pp. 593-599. http://dx.doi.org/10.1111/j.1442-200X.2007.02407.x

[3] C. C. Araujo, M. C. Lima and G. H. Falbo, "Percutaneous Subclavian Central Venous Catheterization in Children and Adolescents: Success, Complications and Related Factors," Jornal de Pediatria, Vol. 83, No. 1, 2007, pp. 64-70.

http://dx.doi.org/10.1111/j.1442-200X.2007.02407.x

[4] A. Citak, M. Karabocuoglu, R. Ucsel and N. Uzel, "Central Venous Catheters in Pediatric Patients-Subclavian Venous Approach as the First Choice," Pediatrics International, Vol. 44, No. 1, 2002, pp. 83-86. http://dx.doi.org/10.1046/j.1442-200X.2002.01509.x

[5] J. Casado-Flores, J. Barja, R. Martino, A. Serrano and Valdivielso, "A Complications of Central Venous Catheterization in Critically Ill Children," Pediatric Critical Care Medicine, Vol. 2, No. 1, 2001, pp. 57-62. http://dx.doi.org/10.1097/00130478-200101000-00012

[6] J. Y. Lefrant, L. Muller, J. E. De La Coussaye, M. Prudhomme, J. Ripart, C. Gouzes, P. Peray, G. Saissi and J. J. Elrdjam, "Risk Factors of Failure and Immediate Complication of SCV Catheterization in Critically Ill Patients," Intensive Care Medicine, Vol. 28, No. 8, 2002, pp. 1036-1041. http://dx.doi.org/10.1007/s00134-002-1364-9

[7] J. Casado-Flores, A. Valdivielso-Serna, L. Perez-Jurado, J. Pozo-Roman, M. Monleon-Luque, J. Garcia-Perez, A. Ruiz-Beltran and M. A. Garcia-Teresa, "SCV Catheterization in Critically Ill Children: Analysis of 322 Cannulations," Intensive Care Medicine, Vol. 17, No. 6, 1991, pp. 350-354.

[8] E. M. Johnson, D. A. Saltzman, G. Suh, R. A. Dahms and A. S. Leonard, "Complications and Risks of Central Venous Catheter Placement in Children," Surgery, Vol. 124, No. 5, 1998, pp. 911-916.

http://dx.doi.org/10.1016/S0039-6060(98)70016-9

[9] T. Pirotte and F. Veyckemans, "Ultrasound-Guided Subclavian Vein Cannulation in Infants and Children: A Novel Approach," British Journal of Anaesthesia, Vol. 98, No. 4, 2007, pp. 509-514. http://dx.doi.org/10.1093/bja/aem041

[10] O. Rhondali, R. Attof, S. Combet, D. Chassard and M. de Queiroz Siqueira, "Ultrasound-Guided SCV Cannulation in Infants: Supraclavicular Approach," Pediatric Anesthesia, Vol. 21, No. 11, 2011, pp. 1136-1141. http://dx.doi.org/10.1111/j.1460-9592.2011.03614.x 